\title{
Glucose-Sparing Action of Ketones Boosts Functions Exclusive to Glucose in the Brain
}

\author{
Yuri Zilberter, ${ }^{1,3}$ and Tanya Zilberter ${ }^{2}$
}

https://doi.org/10.1523/ENEURO.0303-20.2020

${ }^{1}$ Institut de Neurosciences des Systèmes, Aix-Marseille Universite, Institut National de la Santé et de la Recherche Médicale Unité Mixte de Recherche 1106, Marseille 13385, France, ${ }^{2}$ Infotonic Consultancy, Marseille 13009, France, and ${ }^{3}$ Institute of Theoretical and Experimental Biophysics, Russian Academy of Sciences, 142290, Pushchino, Russia

\begin{abstract}
The ketogenic diet (KD) has been successfully used for a century for treating refractory epilepsy and is currently seen as one of the few viable approaches to the treatment of a plethora of metabolic and neurodegenerative diseases. Empirical evidence notwithstanding, there is still no universal understanding of KD mechanism (s). An important fact is that the brain is capable of using ketone bodies for fuel. Another critical point is that glucose's functions span beyond its role as an energy substrate, and in most of these functions, glucose is irreplaceable. By acting as a supplementary fuel, ketone bodies may free up glucose for its other crucial and exclusive function. We propose that this glucose-sparing effect of ketone bodies may underlie the effectiveness of KD in epilepsy and major neurodegenerative diseases, which are all characterized by brain glucose hypometabolism.
\end{abstract}

Key words: aerobic glycolysis; antioxidant system; glucose metabolism; glycogen; ketogenic diet; ketosis

\section{Significance Statement}

The ketogenic diet (KD) was created in the 1920s as a therapy for refractory epilepsy. Since then, evidence accumulated showing its potential for other major neurodegenerative disorders. The exact mechanism of KD's protective activity still remains unknown, nonetheless. In the brain, ketone bodies can be used for cellular energy, at least partially substituting glucose as brain fuel. However, glucose has essential functions beyond those of just energy supply that cannot be provided by alternative substrates. We propose that the glucose-sparing effect of ketone bodies may underlie the effectiveness of KD in epilepsy and other major neurodegenerative diseases which are all characterized by brain glucose hypometabolism.

\section{Introduction}

There is no universally accepted definition of ketogenic diets (KDs). Moreover, there is a tendency to relax previous

Received July 8, 2020; accepted August 27, 2020; First published November 9, 2020.

The authors declare no competing financial interests.

Acknowledgements: This study was supported by the RSF Grant 20-6546035 to $Y . Z$.

Correspondence should be addressed to Yuri Zilberter at yuri.zilberter@ univ-amu.fr.

https://doi.org/10.1523/ENEURO.0303-20.2020

Copyright () 2020 Zilberter and Zilberter

This is an open-access article distributed under the terms of the Creative Commons Attribution 4.0 International license, which permits unrestricted use, distribution and reproduction in any medium provided that the original work is properly attributed. quantitative criteria (Zilberter and Zilberter, 2018) introduced almost a century ago (Wilder and Winter, 1922) that were based on macronutrient composition. Perhaps the most practical notion of whether a diet can be considered to be ketogenic is made by Seyfried (2012): it is "as long as the individual has reduced blood glucose and is producing ketones." Although protein is included in the ketogenic ratio equation (Shaffer, 1921), glucose, ketone bodies, and their interplay determine the dominating metabolic mode: whether the predominant energy supply is glucose or ketone bodies (Westman et al., 2003). The current data allow us to conclude that the functional interaction between glucose and ketone bodies is not a binary winner-take-all process. Here, we attempt to describe more intricate relationships between them. 


\section{Glycolytic ATP Production}

In the case of acute energy demand such as during intense network activity, the brain is able to intensify glycolytic ATP production for rapid supply. Aerobic glycolysis (when glucose is partially converted to lactate in the presence of oxygen, producing two ATP molecules) is disproportionate to the oxygen consumption of glucose utilization when the oxygen delivery is adequate (Dienel and Cruz, 2016). In the resting brain, glycolysis and oxidative phosphorylation rates appear well-matched, indicating nearly complete oxidation of glucose. However, in the activated brain at physiological conditions, e.g., during sensory stimulation and mental testing, aerobic glycolysis has been observed in various brain regions (Dienel and Cruz, 2016; Dienel, 2019a). The cellular contribution to aerobic glycolysis is yet unclear and is a matter of debate (Dienel and Cruz, 2016; Barros et al., 2020). As lactate (in addition to pyruvate) is the end-product of the glycolytic pathway during aerobic glycolysis, it can either be cleared out from the brain or partially consumed as supplementary fuel for neurons (Pellerin and Magistretti, 2012; Dienel and Cruz, 2016). Indeed, aerobic glycolysis as an ATP production mechanism, while lowcapacity, is rapid, ensuring fast ATP resupply in neurons where the dynamic demand for energy is highest (Yellen, 2018). The notion of fast ATP synthesis (aerobic glycolysis) is supported by an immediate rise in extracellular lactate and reduction in glucose levels during brief visual stimulation coincident with spiking activity (Li and Freeman, 2015).

Neurons are rich in mitochondria, a major source of ATP (Hall et al., 2012). However, for some of the neuronal functions, aerobic glycolysis may be the preferential method of fuel generation, for instance, for fast axonal vesicle transport (Zala et al., 2013). Although synaptic transmission is a very energy-demanding process, many presynaptic nerve terminals lack mitochondria (Devine and Kittler, 2018; Tourigny et al., 2019), although mitochondria can migrate to and/or ATP can diffuse into the presynaptic boutons during enhanced synaptic activity (Chamberlain and Sheng, 2019; Rossi and Pekkurnaz, 2019). Recent studies indicate that presynaptic transmission is dependent on activity-induced glycolysis (Ashrafi and Ryan, 2017), while presynapses can use ATP produced by both glycolysis and oxidative phosphorylation to sustain neurotransmission (Ashrafi and Ryan, 2017; Chamberlain and Sheng, 2019; Rossi and Pekkurnaz, 2019). Moreover, dendritic spines that receive most excitatory synaptic inputs have been shown to contain no mitochondria (Kasthuri et al., 2015), despite being sites of intense energy utilization. Although some ATP might diffuse to spine heads from nearby dendritic mitochondria, it is conceivable that spines are reliant on glycolysis instead (Kasthuri et al., 2015). Glycolytic ATP alone, with its limited production capacity, is unlikely to be sufficient to power ion pumping required for the maintenance of ion gradient and membrane potential (Hall et al., 2012), but it may nevertheless play an important role in fast refueling. For instance, the preferential role of glycolytic energy supply was demonstrated for $\mathrm{Na} / \mathrm{K}-\mathrm{ATP}$ ase in fast-twitch skeletal muscle (Okamoto et al., 2001) and cardiac Purkinje cells (Glitsch and Tappe, 1993). Aerobic glycolysis during KD has not been studied yet. However, unlike glucose, ketones cannot be involved in aerobic glycolysis, and thus they contribute to ATP production via oxidative phosphorylation only (Cunnane et al., 2020).

\section{The Cytoplasmic Antioxidant System}

Reactive oxygen species (ROS) in brain cells originate from multiple sources and most of them are generated as byproducts of metabolic reactions. Intracellular ROS come mainly from NADPH oxidase (NOX), xanthine oxidase, nitric oxide synthase, and mitochondria. NOX is the only enzyme with the primary function of generating ROS (Bedard and Krause, 2007; Koju et al., 2019) that are mostly used for the "host defense" (e.g., microbial killing) in organisms. NOX enzymes are predominantly expressed in the cellular plasma membrane (Ma et al., 2017). The enzyme consists of a membrane-bound catalytic core and several cytosolic regulatory subunits (Bedard and Krause, 2007). There are seven known isoforms of NOX with NOX1, NOX2, and NOX4 expressed in multiple brain regions including the cerebral cortex, hippocampus, cerebellum, hypothalamus, midbrain, and/or striatum (Hou et al., 2020). These NOX variants are the most prominent isoforms detected in a variety of brain cell types (Cahill-Smith and Li, 2014; Rastogi et al., 2016; Hou et al., 2020), with NOX2 the dominant form expressed by microglia, neurons, and astrocytes. Activation of NOX results in an increase of extracellular $\mathrm{H}_{2} \mathrm{O}_{2}$ levels followed by $\mathrm{H}_{2} \mathrm{O}_{2}$ entry into the cells via aquaporins (Bienert and Chaumont, 2014). Under "resting conditions" in the brain, NOX is normally dormant and therefore its contribution to the total cellular ROS production under resting or physiological conditions is not clear (Brown and Borutaite, 2012). In pathology, when NOX is activated by specific stimulation (Rastogi et al., 2016), its cytosolic subunits translocate to the membrane and associate to the functioning complex. Under these conditions, mitochondria and NOX are the major ROS producers (Tarafdar and Pula, 2018; Barua et al., 2019). However, before its diffusion to the cytoplasm, the internal consumption of $\mathrm{H}_{2} \mathrm{O}_{2}$ in mitochondria is much higher than originally anticipated (Munro and Pamenter, 2019) and during oxidative stress, mitochondria may be victims rather than producers of oxidative damage (Gandhi and Abramov, 2012). Indeed, in AD models, the effects of mitochondrial ROS were found to be much smaller compared with those of NOX-produced ROS (Angelova and Abramov, 2018).

In the cytoplasm, an efficient anti-oxidative system based largely on the glycolysis-associated pentose-phosphate pathway strictly regulates ROS levels to avoid any oxidative injury (Bolaños and Almeida, 2010; Franco et al., 2019; Cherkas et al., 2020). Thus, brain cells use glucose as an energy provider as well as the substrate for cytoplasmic ROS detoxification mechanisms. The combination of ROS overproduction with inadequate antioxidant defenses (such as when glucose utilization is inhibited) results in oxidative stress and consequent damages, e.g., neural cell death and neurodegeneration (Avery, 2011; Tarafdar and Pula, 2018).

An extreme example of the importance of glucosebased antioxidant defense for cellular function was 
reported by us when glucose in artificial CSF (ACSF) was exchanged for pyruvate in hippocampal slices (Malkov et al., 2014, 2019). The substitution resulted in oxidative stress leading to massive network depolarization (analogous to spreading depression; Pietrobon and Moskowitz, 2014) together with a "metabolic collapse." Importantly, we obtained similar results replacing glucose with other mitochondrial fuels such as lactate or $\beta$-hydroxybutyrate (unpublished), indicating again the unique importance of glucose.

KD improves antioxidant defense by stimulating the endogenous antioxidant system. Mild oxidative stress following KD onset drives nuclear translocation of transcription factor Nrf2, leading to increased synthesis of glutathione which is one of the major components of the antioxidant defense (Pinto et al., 2018; Camberos-Luna and Massieu, 2020).

\section{Glycogen Production in Astrocytes}

The major energy reserve in the brain is glycogen, a macromolecular storage form of glucose (Dienel, 2019b). Found mostly in astrocytes (Dienel and Carlson, 2019; neurons can store less significant amounts; Saez et al., 2014), glycogen is a dynamic participant in brain activity and is regulated by neurotransmitters. Dysregulation of glycogen turnover may cause severe consequences, e.g., Lafora disease with progressive neurodegeneration and epilepsy leading to death in early adulthood (Duran et al., 2019). During intense brain activity, glycogen converted to lactate and released from astrocytes can be used by neurons as mitochondrial fuel. Meanwhile, rapid glycogenolytic generation of ATP may be important for astrocytic energy demands (Dienel and Rothman, 2019), and thus glycogenolysis, by reducing the astrocytic requirement for blood-borne glucose, can spare an equivalent amount of glucose for neuronal utilization (Dienel, 2019a; DiNuzzo et al., 2019). The estimated glucose equivalent of glycogen concentration in astrocytes is up to $40-100 \mathrm{mM}$. Considering the high rate of glycogenolysis, the amount of glucose that can be released via glycogen breakdown is very significant (Dienel, 2019a; DiNuzzo et al., 2019). Unfortunately, glycogen levels in the human brain during KD have yet to be investigated. In rats, one study reported increased inbound glucose (reflecting glycogen content; DeVivo et al., 1978) under KD, while no change in glycogen content was found in another study (Al-Mudallal et al., 1995) or glycogen was decreased (Bough et al., 2006). Carbohydrate intake does not affect brain glycogen content, while in both muscle and liver it does significantly correlate with glycogen levels (Soya et al., 2018).

\section{Production of Major Neurotransmitters}

Glucose fuels de novo synthesis of major neurotransmitters. Precursors for glutamate or GABA are synthesized by astrocytes and then transferred to neurons in the glutamine-glutamate/GABA cycle (Dienel, 2019a). The transmitters released during synaptic activity are transported back to astrocytes, where a fraction ( $\sim 25 \%)$ is degraded with the remainder released and reused by the neurons. The process is very intense, consuming up to
$75 \%$ of the entire glucose consumption in the cortex (Schousboe et al., 2013; Hertz and Rothman, 2016). Glucose metabolism is required for the synthesis of glutamate from glutamine in glutamatergic neurons (Bak et al., 2006; Lund et al., 2009; Chowdhury et al., 2014; Hertz and Rothman, 2016), but in GABAergic neurons, $\beta$-hydroxybutyrate is capable of replacing glucose for GABA production (Hertz and Rothman, 2016).

\section{Glucose Utilization in the Brain during Ketosis}

The KD is an efficient clinical treatment used for over a century to decrease brain hyperexcitability and seizures via a yet unclear mechanism. Carbohydrate limitation in KD led to a popular notion that glycolysis inhibition may partially reproduce the effects of KD. However, this hypothesis ignores the important fact that glycolysis inhibition a priori results in energy deprivation; meanwhile, no energy deficiency occurs under KD [in rats on KD, the brain ATP level was found either unchanged (Al-Mudallal et al., 1996; Bough et al., 2006) or increased (DeVivo et al., 1978; Nakazawa et al., 1983)]. However, do ketones inhibit glycolysis [as, for instance, does 2-deoxy-D-glucose (Pajak et al., 2019) or iodoacetate (Schmidt and Dringen, 2009)] or do they just compete with glucose as mitochondrial fuel? The NMR study of Valente-Silva et al. (2015) is often cited as evidence of glycolysis inhibition. There are a number of important flaws in this study, however: (1) the $400-\mu \mathrm{m}$-thick brain slices were superfused with oxygenated ACSF at $3 \mathrm{ml} / \mathrm{min}$ rate that results in anoxic conditions within the tissue (Ivanov and Zilberter, 2011), making oxidative phosphorylation impossible; and (2) the authors used 4-AP to induce network activity, while epileptiform activity induced by 4-AP was itself shown to be a strong inhibitor of glycolysis (Malkov et al., 2018). To the best of our knowledge, there are no other reports on ketones' direct inhibitory action on glycolysis.

There have been many attempts to estimate brain glucose utilization under KD, with variable results. In awake animals, glucose utilization either increased (Yudkoff et al., 2005), did not change (Al-Mudallal et al., 1995) or decreased (Melø et al., 2006), while brain glucose levels either increased (Melø et al., 2006), did not change (DeVivo et al., 1978; Yudkoff et al., 2005) or decreased (Samala et al., 2011). Utilization of the fluorodeoxyglucose $\left({ }^{18} \mathrm{~F}\right)$-positron emission tomography (FDG-PET) technique in animals requires anesthesia that introduces a brain state quite different from an awake one. Nevertheless, under anesthesia, glucose utilization either increased (Pifferi et al., 2011; Roy et al., 2012), remained unchanged (Yudkoff et al., 2005), or decreased (LaManna et al., 2009; Zhang et al., 2013). The variability of the results may be explained by the presence of confounding factors such as, e.g., the use of anesthesia, significant age difference and the relatively high interindividual variation in plasma ketones during KD (Pifferi et al., 2011; Roy et al., 2012).

Interestingly, in healthy young/middle age humans, cerebral glucose utilization evaluated by arteriovenous difference was decreased under acute hyperketonemia (a blood infusion of $\beta$-hydroxybutyrate; Hasselbalch et al., 


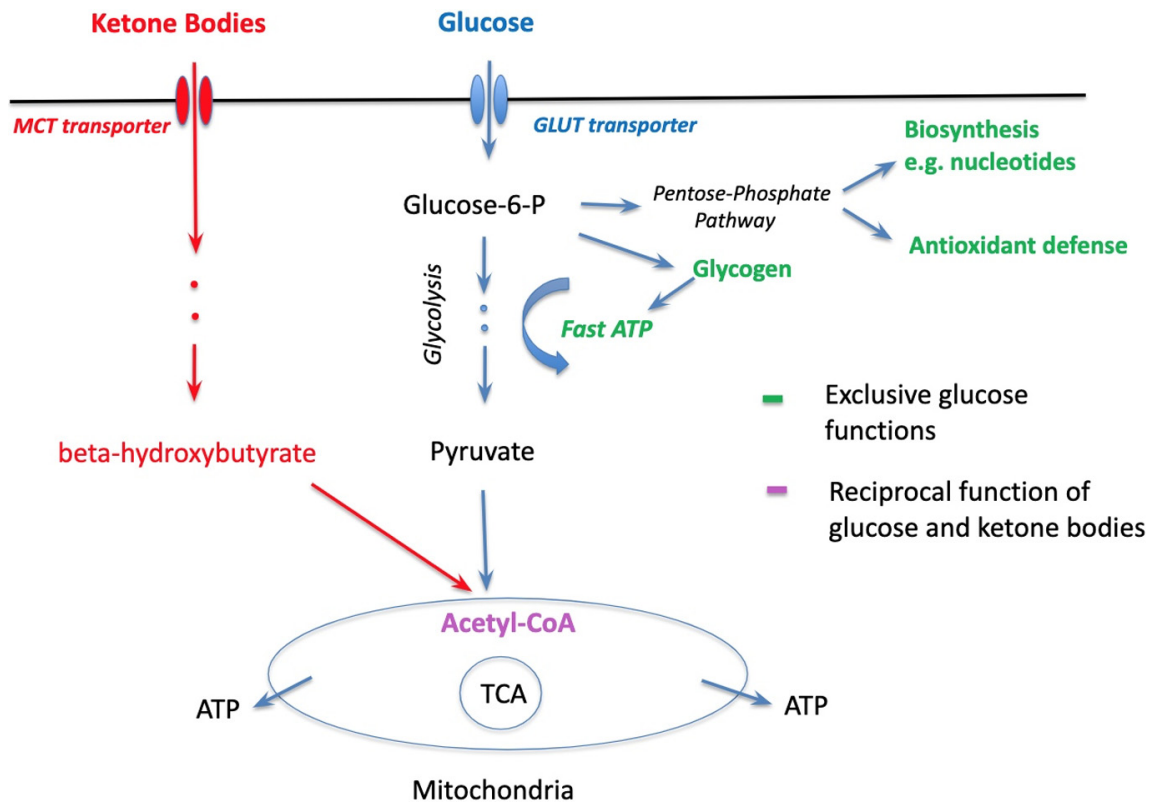

Figure 1. Roles of glucose and ketone bodies in brain cells: parallel, converging, and exclusive. Red: ketone bodies' pathway to mitochondria. Blue: glucose pathway to mitochondria. Purple: the point of convergence. Green: exclusive roles of glucose.

1996) or following $3 \mathrm{~d}$ of fasting (Hasselbalch et al., 1994). Recent studies using the FDG-PET technique confirmed these results reporting decreased brain glucose utilization in healthy humans under KD (Courchesne-Loyer et al., 2017) or acute hyperketonemia (Svart et al., 2018). Importantly and however, similar recordings during ketogenic intervention in humans suffering mild cognitive impairment (Fortier et al., 2019) or mild-moderate Alzheimer's disease (AD; Croteau et al., 2018b) revealed no change in brain glucose utilization, while brain ketone metabolism was found to be normal (Castellano et al., 2015; Croteau et al., 2018a). Notably, glucose hypometabolism is a hallmark of $A D$ pathogenesis (Caminiti et al., 2018; Gordon et al., 2018; Butterfield and Halliwell, 2019). Indeed, disrupted glucose metabolism associated with oxidative stress is the common feature of major neurodegenerative diseases (Zilberter and Zilberter, 2017; Cunnane et al., 2020; Tang, 2020) and epilepsy (Pearson-Smith and Patel, 2017; Zilberter and Zilberter, 2017; Patel, 2018). For instance, AD pathology occurs well before (up to two decades prior) the onset of clinical symptoms (Caminiti et al., 2018; Gordon et al., 2018; Butterfield and Halliwell, 2019) with dysfunctional glucose metabolism as one earliest manifestation. In human epilepsy, clinical tests using FDG-PET imaging have established that decreased brain glucose utilization during quiescent (interictal) periods is a widely recognized biomarker of epileptogenesis (Sarikaya, 2015; Lotan et al., 2020). Since glucose utilization underlies vital brain functions such as energy supply and antioxidant defense (see above), it is not surprising that disturbances in glucose metabolism can lead to a chain of harmful consequences, and thus likely represent a major underlying cause of disease initiation and progression (Pearson-Smith and Patel, 2017; Zilberter and Zilberter, 2017; Butterfield and Halliwell, 2019). Therefore, as also suggested previously (Cunnane et al., 2016a; Croteau et al., 2018b), it is logical to conclude that in a normal brain, where glucose can fully cover energy needs, the addition of ketones as mitochondrial fuel competitors reduces the need for a part of glucose function and the total glucose utilization goes down. In pathology, when glucose utilization is impaired leading to energy deficiency, ketone bodies compensate at least partially this energy gap, leaving more glucose available for its other vital functions such as mentioned above.

In fact, the glucose-sparing effect may be dominant in ketone's beneficial therapeutic function ensemble. Clinical effects of KD are mostly known regarding epilepsy as childhood epilepsy has been successfully treated with KD since the 1920s (Wilder, 1921). Recently, however, the KD began to be used in preclinical studies of other disorders, including neurodegenerative, psychiatric, and brain injury (McDonald and Cervenka, 2018; Camberos-Luna and Massieu, 2020; Kraeuter et al., 2020), demonstrating promising efficiency that has also been confirmed in clinical trials in mild cognitive impairment, $A D$, and Parkinson's disease patients (for review, see Cunnane et al., 2020). Meanwhile, it has been noted that the efficacy of KD in an array of disorders with distinct pathophysiologies may indicate shared underlying pathologic mechanisms (Kraeuter et al., 2020). In the case of major neurodegenerative diseases, one such mechanism is glucose hypometabolism (Zilberter and Zilberter, 2017; Cunnane et al., 2020; Tang, 2020). Definitely, impaired glucose metabolism may lead to energy deficiency that ketones can partially compensate by boosting mitochondrial oxidation. Here, it is interesting to note that in the case of early AD stages, neither cerebral oxygen consumption (Hoyer et al., 1988; Hoyer, 1992) nor mitochondrial ability for ketone utilization changed (Castellano et al., 2015; Croteau et al., 2018a), suggesting normal mitochondrial functioning (Cunnane et al., 2016b, 
2020), despite pronounced glucose hypometabolism. Indeed, we demonstrated recently in hippocampal slices that amyloid- $\beta$ (Malkov et al., 2020) as well as epileptiform activity (Malkov et al., 2018) strongly inhibited glycolysis while mitochondrial function remained normal or even upregulated, potentially as a compensatory mechanism. This therefore suggests that glucose metabolism deficiency mostly affects functions not related to oxidative phosphorylation and thus ketones possibly leave more glucose available for other vital functions (Fig. 1) that may be especially important for the therapeutic effects of KD. The potential importance of the glucose-sparing effect of ketones has been noted previously in reports considering neurodegenerative diseases (Cunnane et al., 2016a, 2020).

\section{Conclusions}

Glucose does not share some of its functions in the brain with other metabolic substrates, which makes it an exclusive neurometabolite. The frequent claim that ketones directly inhibit the process of glycolysis is not supported by experimental evidence and seems theoretically unlikely. Moreover, glycolysis inhibition modifies a number of cellular functions that lead to unpredictable variations of network excitability, and in the long-run has been shown to result in epileptogenesis (Samokhina et al., $2017,2020)$. In pathology, ketones are capable of partially taking over the glucose's energy fuel role in mitochondria and presumably spare glucose for its other exclusive functions. Here, we summarized the evidence and offer a non-antagonistic view of the ketone bodies-glucose interplay during metabolic shifting. The glucose-sparing effect of ketone bodies may determine the efficiency of the ketogenic regime and is especially important in epilepsy and major neurodegenerative diseases characterized by significantly impaired brain glucose utilization.

\section{References}

Al-Mudallal AS, Levin BE, Lust WD, Harik SI (1995) Effects of unbalanced diets on cerebral glucose metabolism in the adult rat. Neurology 45:2261-2265.

Al-Mudallal AS, LaManna JC, Lust WD, Harik SI (1996) Diet-induced ketosis does not cause cerebral acidosis. Epilepsia 37:258-261.

Angelova PR, Abramov AY (2018) Role of mitochondrial ROS in the brain: from physiology to neurodegeneration. FEBS Lett 592:692702.

Ashrafi G, Ryan TA (2017) Glucose metabolism in nerve terminals. Curr Opin Neurobiol 45:156-161.

Avery SV (2011) Molecular targets of oxidative stress. Biochem J 434:201-210.

Bak LK, Schousboe A, Sonnewald U, Waagepetersen HS (2006) Glucose is necessary to maintain neurotransmitter homeostasis during synaptic activity in cultured glutamatergic neurons. J Cereb Blood Flow Metab 26:1285-1297.

Barros LF, Felipe Barros L, Ruminot I, Martín AS, Lerchundi R, Fernández-Moncada I, Baeza-Lehnert F (2020) Aerobic glycolysis in the brain: Warburg and Crabtree contra Pasteur. Neurochem Res. Advance online publication. Retrieved Jan 24, 2020. doi: 10.1007/s11064-020-02964-w.

Barua S, Kim JY, Yenari MA, Lee JE (2019) The role of NOX inhibitors in neurodegenerative diseases. IBRO Rep 7:59-69.
Bedard K, Krause KH (2007) The NOX family of ROS-generating NADPH oxidases: physiology and pathophysiology. Physiol Rev 87:245-313.

Bienert GP, Chaumont F (2014) Aquaporin-facilitated transmembrane diffusion of hydrogen peroxide. Biochim Biophys Acta 1840:1596-1604.

Bolaños JP, Almeida A (2010) The pentose-phosphate pathway in neuronal survival against nitrosative stress. IUBMB Life 62:14-18.

Bough KJ, Wetherington J, Hassel B, Pare JF, Gawryluk JW, Greene JG, Shaw R, Smith Y, Geiger JD, Dingledine RJ (2006) Mitochondrial biogenesis in the anticonvulsant mechanism of the ketogenic diet. Ann Neurol 60:223-235.

Brown GC, Borutaite V (2012) There is no evidence that mitochondria are the main source of reactive oxygen species in mammalian cells. Mitochondrion 12:1-4.

Butterfield DA, Halliwell B (2019) Oxidative stress, dysfunctional glucose metabolism and Alzheimer disease. Nat Rev Neurosci $20: 148-160$.

Cahill-Smith S, Li JM (2014) Oxidative stress, redox signalling and endothelial dysfunction in ageing-related neurodegenerative diseases: a role of NADPH oxidase 2. Br J Clin Pharmacol 78:441453.

Camberos-Luna L, Massieu L (2020) Therapeutic strategies for ketosis induction and their potential efficacy for the treatment of acute brain injury and neurodegenerative diseases. Neurochem Int 133:104614.

Caminiti SP, Ballarini T, Sala A, Cerami C, Presotto L, Santangelo R, Fallanca F, Vanoli EG, Gianolli L, lannaccone S, Magnani G, Perani D, Parnetti L, Eusebi P, Frisoni G, Nobili F, Picco A, Scarpini E, BIOMARKAPD Project (2018) FDG-PET and CSF biomarker accuracy in prediction of conversion to different dementias in a large multicentre $\mathrm{MCl}$ cohort. Neuroimage Clin 18:167-177.

Castellano CA, Nugent S, Paquet N, Tremblay S, Bocti C, Lacombe G, Imbeault H, Turcotte E, Fulop T, Cunnane SC (2015) Lower brain 18F-fluorodeoxyglucose uptake but normal 11C-acetoacetate metabolism in mild Alzheimer's disease dementia. J Alzheimers Dis 43:1343-1353.

Chamberlain KA, Sheng ZH (2019) Mechanisms for the maintenance and regulation of axonal energy supply. J Neurosci Res 97:897913.

Cherkas A, Holota S, Mdzinarashvili T, Gabbianelli R, Zarkovic N (2020) Glucose as a major antioxidant: when, what for and why it fails? Antioxidants (Basel) 9:140.

Chowdhury GMI, Jiang L, Rothman DL, Behar KL (2014) The contribution of ketone bodies to basal and activity-dependent neuronal oxidation in vivo. J Cereb Blood Flow Metab 34:1233-1242.

Courchesne-Loyer A, Croteau E, Castellano CA, St-Pierre V, Hennebelle M, Cunnane SC (2017) Inverse relationship between brain glucose and ketone metabolism in adults during short-term moderate dietary ketosis: a dual tracer quantitative positron emission tomography study. J Cereb Blood Flow Metab 37:2485-2493.

Croteau E, Castellano CA, Fortier M, Bocti C, Fulop T, Paquet N, Cunnane SC (2018a) A cross-sectional comparison of brain glucose and ketone metabolism in cognitively healthy older adults, mild cognitive impairment and early Alzheimer's disease. Exp Gerontol 107:18-26.

Croteau E, Castellano C-A, Richard MA, Fortier M, Nugent S, Lepage M, Duchesne S, Whittingstall K, Turcotte ÉE, Bocti C, Fülöp T, Cunnane SC (2018b) Ketogenic medium chain triglycerides increase brain energy metabolism in Alzheimer's disease. $\mathrm{J}$ Alzheimers Dis 64:551-561.

Cunnane SC, Courchesne-Loyer A, St-Pierre V, Vandenberghe C, Pierotti T, Fortier M, Croteau E, Castellano CA (2016a) Can ketones compensate for deteriorating brain glucose uptake during aging? Implications for the risk and treatment of Alzheimer's disease. Ann NY Acad Sci 1367:12-20.

Cunnane SC, Vandenberghe C, Fortier M, Hennebelle M, Croteau E, Bocti C, Fulop T, Castellano CA (2016b) Can ketones help rescue brain fuel supply in later life? Implications for cognitive health 
during aging and the treatment of Alzheimer's disease. Front Mol Neurosci 9:53.

Cunnane SC, Trushina E, Morland C, Prigione A, Casadesus G, Andrews ZB, Beal MF, Bergersen LH, Brinton RD, de la Monte S, Eckert A, Harvey J, Jeggo R, Jhamandas JH, Kann O, la Cour CM, Martin WF, Mithieux G, Moreira PI, Murphy MP, et al. (2020) Brain energy rescue: an emerging therapeutic concept for neurodegenerative disorders of ageing. Nat Rev Drug Discov 19:609-633.

Devine MJ, Kittler JT (2018) Mitochondria at the neuronal presynapse in health and disease. Nat Rev Neurosci 19:63-80.

DeVivo DC, Leckie MP, Ferrendelli JS, McDougal DB Jr (1978) Chronic ketosis and cerebral metabolism. Ann Neurol 3:331-337.

Dienel GA (2019a) Brain glucose metabolism: integration of energetics with function. Physiol Rev 99:949-1045.

Dienel GA (2019b) Does shuttling of glycogen-derived lactate from astrocytes to neurons take place during neurotransmission and memory consolidation? J Neurosci Res 97:863-882.

Dienel GA, Cruz NF (2016) Aerobic glycolysis during brain activation: adrenergic regulation and influence of norepinephrine on astrocytic metabolism. J Neurochem 138:14-52.

Dienel GA, Carlson GM (2019) Major advances in brain glycogen research: understanding of the roles of glycogen have evolved from emergency fuel reserve to dynamic, regulated participant in diverse brain functions. Adv Neurobiol 23:1-16.

Dienel GA, Rothman DL (2019) Glycogenolysis in cerebral cortex during sensory stimulation, acute hypoglycemia, and exercise: impact on astrocytic energetics, aerobic glycolysis, and astrocyteneuron interactions. Adv Neurobiol 23:209-267.

DiNuzzo M, Walls AB, Öz G, Seaquist ER, Waagepetersen HS, Bak LK, Nedergaard M, Schousboe A (2019) State-dependent changes in brain glycogen metabolism. Adv Neurobiol 23:269-309.

Duran J, Gruart A, López-Ramos JC, Delgado-García JM, Guinovart JJ (2019) Glycogen in astrocytes and neurons: physiological and pathological aspects. Adv Neurobiol 23:311-329.

Fortier M, Castellano CA, Croteau E, Langlois F, Bocti C, St-Pierre V, Vandenberghe C, Bernier M, Roy M, Descoteaux M, Whittingstall K, Lepage M, Turcotte ÉE, Fulop T, Cunnane SC (2019) A ketogenic drink improves brain energy and some measures of cognition in mild cognitive impairment. Alzheimers Dement 15:625-634.

Franco R, Navarro G, Martínez-Pinilla E (2019) Antioxidant defense mechanisms in erythrocytes and in the central nervous system. Antioxidants (Basel) 8:46.

Gandhi S, Abramov AY (2012) Mechanism of oxidative stress in neurodegeneration. Oxid Med Cell Longev 2012:428010.

Glitsch HG, Tappe A (1993) The Na+/K+ pump of cardiac Purkinje cells is preferentially fuelled by glycolytic ATP production. Pflugers Arch 422:380-385.

Gordon BA, Blazey TM, Su Y, Hari-Raj A, Dincer A, Flores S, Christensen J, McDade E, Wang G, Xiong C, Cairns NJ, Hassenstab J, Marcus DS, Fagan AM, Jack CR, Hornbeck RC, Paumier KL, Ances BM, Berman SB, Brickman AM, et al. (2018) Spatial patterns of neuroimaging biomarker change in individuals from families with autosomal dominant Alzheimer's disease: a longitudinal study. Lancet Neurol 17:241-250.

Hall CN, Klein-Flügge MC, Howarth C, Attwell D (2012) Oxidative phosphorylation, not glycolysis, powers presynaptic and postsynaptic mechanisms underlying brain information processing. $\mathrm{J}$ Neurosci 32:8940-8951.

Hasselbalch SG, Knudsen GM, Jakobsen J, Hageman LP, Holm S, Paulson OB (1994) Brain metabolism during short-term starvation in humans. J Cereb Blood Flow Metab 14:125-131.

Hasselbalch SG, Madsen PL, Hageman LP, Olsen KS, Justesen N, Holm S, Paulson OB (1996) Changes in cerebral blood flow and carbohydrate metabolism during acute hyperketonemia. Am J Physiol 270:E746-E751.

Hertz L, Rothman DL (2016) Glucose, lactate, $\beta$-hydroxybutyrate, acetate, GABA, and succinate as substrates for synthesis of glutamate and GABA in the glutamine-glutamate/GABA cycle. Adv Neurobiol 13:9-42.
Hou L, Zhang L, Hong J-S, Zhang D, Zhao J, Wang Q (2020) Nicotinamide adenine dinucleotide phosphate oxidase and neurodegenerative diseases: mechanisms and therapy. Antioxid Redox Signal 33:374-393.

Hoyer S (1992) Oxidative energy metabolism in Alzheimer brain. Studies in early-onset and late-onset cases. Mol Chem Neuropathol 16:207-224.

Hoyer S, Oesterreich K, Wagner O (1988) Glucose metabolism as the site of the primary abnormality in early-onset dementia of Alzheimer type? J Neurol 235:143-148.

Ivanov A, Zilberter Y (2011) Critical state of energy metabolism in brain slices: the principal role of oxygen delivery and energy substrates in shaping neuronal activity. Front Neuroenergetics 3:9.

Kasthuri N, Hayworth KJ, Berger DR, Schalek RL, Conchello JA, Knowles-Barley S, Lee D, Vázquez-Reina A, Kaynig V, Jones TR, Roberts M, Morgan JL, Tapia JC, Seung HS, Roncal WG, Vogelstein JT, Burns R, Sussman DL, Priebe CE, Pfister H, et al. (2015) Saturated reconstruction of a volume of neocortex. Cell 162:648-661.

Koju N, Taleb A, Zhou J, Lv G, Yang J, Cao X, Lei H, Ding Q (2019) Pharmacological strategies to lower crosstalk between nicotinamide adenine dinucleotide phosphate (NADPH) oxidase and mitochondria. Biomed Pharmacother 111:1478-1498.

Kraeuter AK, Phillips R, Sarnyai Z (2020) Ketogenic therapy in neurodegenerative and psychiatric disorders: from mice to men. Prog Neuropsychopharmacol Biol Psychiatry 101:109913.

LaManna JC, Salem N, Puchowicz M, Erokwu B, Koppaka S, Flask C, Lee Z (2009) Ketones suppress brain glucose consumption. Adv Exp Med Biol 645:301-306.

Li B, Freeman RD (2015) Neurometabolic coupling between neural activity, glucose, and lactate in activated visual cortex. J Neurochem 135:742-754.

Lotan E, Friedman KP, Davidson T, Shepherd TM (2020) Brain 18FFDG-PET: utility in the diagnosis of dementia and epilepsy. Isr Med Assoc J 22:178-184.

Lund TM, Risa O, Sonnewald U, Schousboe A, Waagepetersen HS (2009) Availability of neurotransmitter glutamate is diminished when beta-hydroxybutyrate replaces glucose in cultured neurons. J Neurochem 110:80-91.

Ma MW, Wang J, Zhang Q, Wang R, Dhandapani KM, Vadlamudi RK, Brann DW (2017) NADPH oxidase in brain injury and neurodegenerative disorders. Mol Neurodegener 12:7.

Malkov A, Ivanov Al, Popova I, Mukhtarov M, Gubkina O, Waseem T, Bregestovski P, Zilberter Y (2014) Reactive oxygen species initiate a metabolic collapse in hippocampal slices: potential trigger of cortical spreading depression. J Cereb Blood Flow Metab 34:1540-1549.

Malkov A, Ivanov Al, Buldakova S, Waseem T, Popova I, Zilberter M, Zilberter Y (2018) Seizure-induced reduction in glucose utilization promotes brain hypometabolism during epileptogenesis. Neurobiol Dis 116:28-38.

Malkov A, Ivanov Al, Latyshkova A, Bregestovski $\mathrm{P}$, Zilberter $\mathrm{M}$, Zilberter Y (2019) Activation of nicotinamide adenine dinucleotide phosphate oxidase is the primary trigger of epileptic seizures in rodent models. Ann Neurol 85:907-920.

Malkov A, Popova I, Ivanov A, Jang SS, Yoon SY, Osypov A, Huang Y, Zilberter $Y$, Zilberter M (2020) A $\beta$-induced NOX2 activation underlies oxidative stress leading to brain hypometabolism and hyperactivity in Alzheimer's disease. bioRxiv. doi: https://doi.org/ 10.1101/2020.08.12.248492.

McDonald TJW, Cervenka MC (2018) Ketogenic diets for adult neurological disorders. Neurotherapeutics 15:1018-1031.

Melø TM, Nehlig A, Sonnewald U (2006) Neuronal-glial interactions in rats fed a ketogenic diet. Neurochem Int 48:498-507.

Munro D, Pamenter ME (2019) Comparative studies of mitochondrial reactive oxygen species in animal longevity: technical pitfalls and possibilities. Aging Cell 18:e13009.

Nakazawa M, Kodama S, Matsuo T (1983) Effects of ketogenic diet on electroconvulsive threshold and brain contents of adenosine nucleotides. Brain Dev 5:375-380. 
Okamoto KEN, Wang W, Rounds JAN, Chambers EA, Jacobs DO (2001) ATP from glycolysis is required for normal sodium homeostasis in resting fast-twitch rodent skeletal muscle. Am J Physiol Endocrinol Metab 281:E479-E488.

Pajak B, Siwiak E, Sołtyka M, Priebe A, Zieliński R, Fokt I, Ziemniak M, Jaśkiewicz A, Borowski R, Domoradzki T, Priebe W (2019) 2Deoxy-d-glucose and its analogs: from diagnostic to therapeutic agents. Int J Mol Sci 21:234.

Patel M (2018) A metabolic paradigm for epilepsy. Epilepsy Curr 18:318-322.

Pearson-Smith JN, Patel M (2017) Metabolic dysfunction and oxidative stress in epilepsy. Int J Mol Sci 18:2365.

Pellerin L, Magistretti PJ (2012) Sweet sixteen for ANLS. J Cereb Blood Flow Metab 32:1152-1166.

Pietrobon D, Moskowitz MA (2014) Chaos and commotion in the wake of cortical spreading depression and spreading depolarizations. Nat Rev Neurosci 15:379-393.

Pifferi F, Tremblay S, Croteau E, Fortier M, Tremblay-Mercier J, Lecomte R, Cunnane SC (2011) Mild experimental ketosis increases brain uptake of $11 \mathrm{C}$-acetoacetate and 18F-fluorodeoxyglucose: a dual-tracer PET imaging study in rats. Nutr Neurosci 14:51-58.

Pinto A, Bonucci A, Maggi E, Corsi M, Businaro R (2018) Anti-oxidant and anti-inflammatory activity of ketogenic diet: new perspectives for neuroprotection in Alzheimer's disease. Antioxidants (Basel) 7:63.

Rastogi R, Geng X, Li F, Ding Y (2016) NOX activation by subunit interaction and underlying mechanisms in disease. Front Cell Neurosci 10:301.

Rossi MJ, Pekkurnaz G (2019) Powerhouse of the mind: mitochondrial plasticity at the synapse. Curr Opin Neurobiol 57:149-155.

Roy M, Nugent S, Tremblay-Mercier J, Tremblay S, CourchesneLoyer A, Beaudoin JF, Tremblay L, Descoteaux M, Lecomte R, Cunnane SC (2012) The ketogenic diet increases brain glucose and ketone uptake in aged rats: a dual tracer PET and volumetric MRI study. Brain Res 1488:14-23.

Saez I, Duran J, Sinadinos C, Beltran A, Yanes O, Tevy MF, Martínez-Pons C, Milán M, Guinovart JJ (2014) Neurons have an active glycogen metabolism that contributes to tolerance to hypoxia. J Cereb Blood Flow Metab 34:945-955.

Samala R, Klein J, Borges K (2011) The ketogenic diet changes metabolite levels in hippocampal extracellular fluid. Neurochem Int 58:5-8.

Samokhina E, Popova I, Malkov A, Ivanov Al, Papadia D, Osypov A, Molchanov M, Paskevich S, Fisahn A, Zilberter M, Zilberter $Y$ (2017) Chronic inhibition of brain glycolysis initiates epileptogenesis. J Neurosci Res 95:2195-2206.

Samokhina E, Malkov A, Samokhin A, Popova I (2020) Selective hippocampal cell damage and mossy fiber sprouting induced by chronic intracerebral injections of 2-deoxy-D-glucose. Gen Physiol Biophys 39:99-106.

Sarikaya I (2015) PET studies in epilepsy. Am J Nucl Med Mol Imaging 5:416-430.

Schmidt MM, Dringen R (2009) Differential effects of iodoacetamide and iodoacetate on glycolysis and glutathione metabolism of cultured astrocytes. Front Neuroenergetics 1:1.
Schousboe A, Bak LK, Waagepetersen HS (2013) Astrocytic control of biosynthesis and turnover of the neurotransmitters glutamate and GABA. Front Endocrinol (Lausanne) 4:102.

Seyfried T (2012) Cancer as a metabolic disease: on the origin, management, and prevention of cancer. Hoboken: Wiley.

Shaffer PA (1921) Antiketogenesis. I. An in vitro analogy. J Biol Chem 47:433.

Soya M, Matsui T, Shima T, Jesmin S, Omi N, Soya H (2018) Hyperhippocampal glycogen induced by glycogen loading with exhaustive exercise. Sci Rep 8:1285.

Svart M, Gormsen LC, Hansen J, Zeidler D, Gejl M, Vang K, Aanerud J, Moeller N (2018) Regional cerebral effects of ketone body infusion with 3-hydroxybutyrate in humans: reduced glucose uptake, unchanged oxygen consumption and increased blood flow by positron emission tomography. A randomized, controlled trial. PLoS One 13:e0190556.

Tang BL (2020) Glucose, glycolysis, and neurodegenerative diseases. J Cell Physiol 235:7653-7662.

Tarafdar A, Pula G (2018) The role of NADPH oxidases and oxidative stress in neurodegenerative disorders. Int J Mol Sci 19:3824.

Tourigny DS, Karim MKA, Echeveste R, Kotter MRN, O'Neill JS (2019) Energetic substrate availability regulates synchronous activity in an excitatory neural network. PLoS One 14:e0220937.

Valente-Silva P, Lemos C, Köfalvi A, Cunha RA, Jones JG (2015) Ketone bodies effectively compete with glucose for neuronal acetyl-CoA generation in rat hippocampal slices. NMR Biomed 28:1111-1116.

Westman EC, Mavropoulos J, Yancy WS, Volek JS (2003) A review of low-carbohydrate ketogenic diets. Curr Atheroscler Rep 5:476483.

Wilder RM (1921) The effects of ketonemia on the course of epilepsy. Mayo Clin Proc 2:307-308.

Wilder RM, Winter MD (1922) The threshold of ketogenesis. J Biol Chem 52:393-401.

Yellen G (2018) Fueling thought: management of glycolysis and oxidative phosphorylation in neuronal metabolism. J Cell Biol 217:2235-2246.

Yudkoff M, Daikhin Y, Nissim I, Horyn O, Lazarow A, Luhovyy B, Wehrli S, Nissim I (2005) Response of brain amino acid metabolism to ketosis. Neurochem Int 47:119-128.

Zala D, Hinckelmann MV, Yu H, Lyra da Cunha MM, Liot G, Cordelières FP, Marco S, Saudou F (2013) Vesicular glycolysis provides on-board energy for fast axonal transport. Cell 152:479491.

Zhang Y, Kuang Y, Xu K, Harris D, Lee Z, LaManna J, Puchowicz MA (2013) Ketosis proportionately spares glucose utilization in brain. J Cereb Blood Flow Metab 33:1307-1311.

Zilberter Y, Zilberter M (2017) The vicious circle of hypometabolism in neurodegenerative diseases: ways and mechanisms of metabolic correction. J Neurosci Res 95:2217-2235.

Zilberter T, Zilberter Y (2018) Ketogenic ratio determines metabolic effects of macronutrients and prevents interpretive bias. Front Nutr 5:75. 